\title{
The relationship between intraoperative blood pressure variability and all-cause mortality and postoperative acute kidney injury
}

Wafa Bellan, M.D. ${ }^{1}$, Walid Saliba, M.D., MPH ${ }^{2,3}$ Nili Stein, MPH ${ }^{2}$ Dmitri Bystritski, M D1, Reuven Pizov, M.D ${ }^{1,3}$ Arieh Eden, M.D. ${ }^{1}$

1. Department of Anesthesiology, Critical Care \& pain Medicine, 2. Department of Community Medicine and Epidemiology, Lady Davis

Carmel Medical Center, \& 3 Ruth and Bruch Rappaport Faculty of Medicine, Technion - Israel Institute of Technology, Haifa, Israel.

Background: The Duration of intraoperative hypotension is associated with increased morbidity (renal injury and ischemic heart disease) and mortality (1). The importance of blood pressure (BP) variability per se is not yet fully understood and has barley been investigated. In other medical fields Decreased variability in various physiologic parameters is considered a sign of disease (2).

Objective: To assess the association between intraoperative blood pressure variability and postoperative acute kidney injury (AKI) and mortality.

Methods: A retrospective cohort study of consecutive adults who underwent surgery at the Lady Davis Carmel Medical Center between 1.1.2005-31.12.2015 of $>120$ minutes duration and had invasive BP monitoring. Operations under local anesthesia and cardiac/vascular surgeries were excluded.

KIDGO stage 1,2 or 3 were defined according to the highest creatinine value measured during the first week after surgery and baseline creatinine (lowest value in 3 months) (3). Intraoperative blood pressure variability was calculated for each patient using the generalized average real variability (ARV) equation. Its association with all-cause mortality and AKI was assessed using Cox proportional hazard regression models.

Generalized MBP Average real variability was calculated for each patient (4):

Generalized ARV $=\frac{1}{T} \sum_{k=1}^{N-1}\left|\mathrm{BP}_{k+1}-\mathrm{BP}_{k}\right| \mathrm{mmHg} / \mathrm{min}$

Results: overall, 7677 patients $(61.2 \%$ men, $83.8 \%$ elective) with mean age $66.1 \pm 13.4$ years were included in the study. Baseline data are presented in table 1. Patients were divided into four quartiles according to their ARV in ascending order (table 2). Thirty days and six months mortality decreased with increasing intra operative BP variability (figure 1 ). The adjusted hazard ratio $(\mathrm{HR})$ for all-cause 30 days mortality was $0.90(95 \% \mathrm{Cl}, 0.67-1.22)$ in group II, $0.76(0.55-$ $1.05)$ in group III, and $0.68(0.49-0.96)$ in group IV which included the patients with highest ARV.

Increase in intraoperative blood pressure variability was associated with an increase in postoperative AKI (table 3 ). This finding was irrespective of the severity of AKI.

Figure 1: Kaplan Meyer curve of Survival according to mean blood pressure variability quartile $(\mathrm{mmHg} / \mathrm{min})$

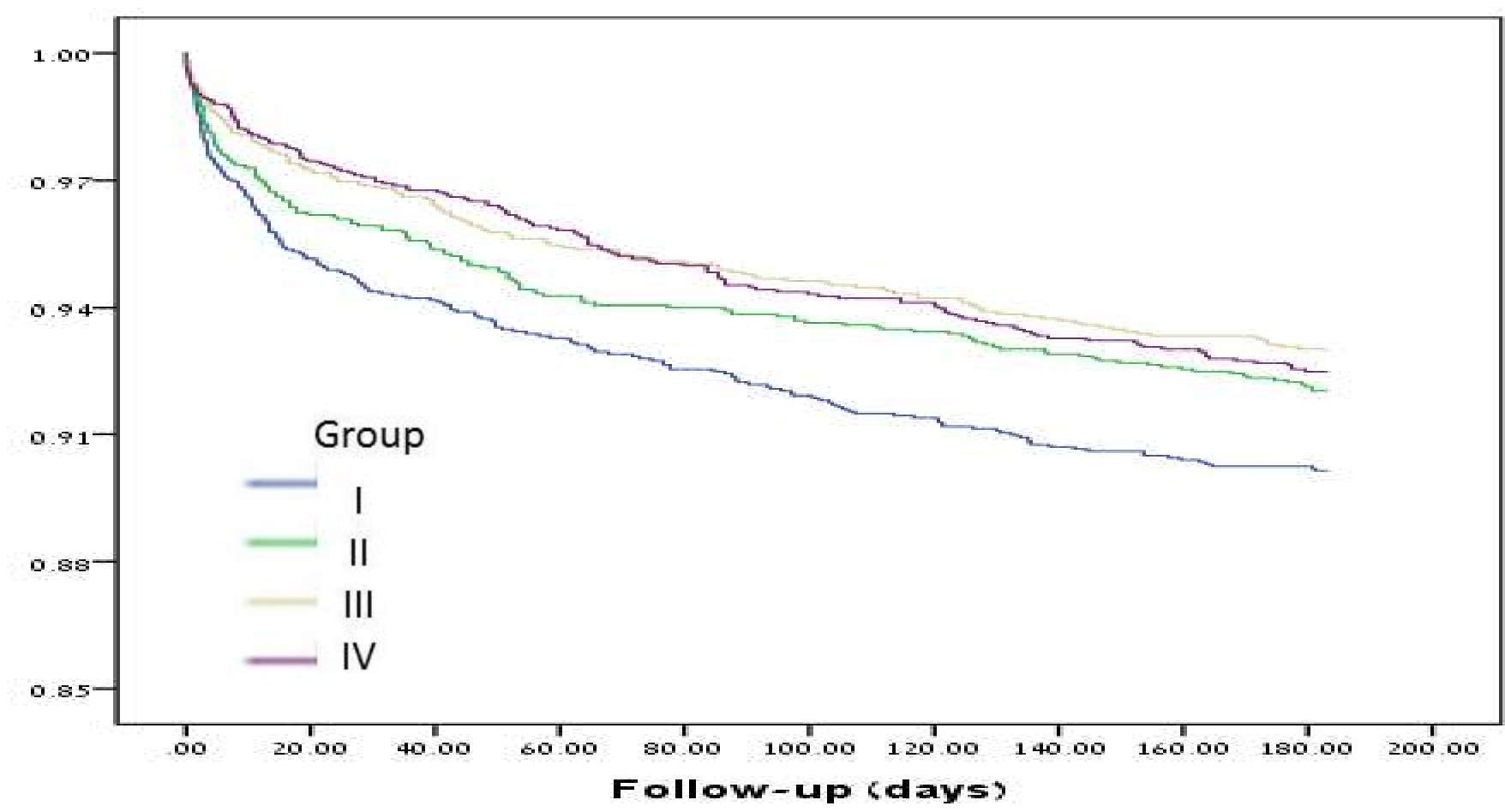

Table 1: Demographics and Baseline characters

ARV: Average real variability, IHD: Ischemic Heart Disease, CHF: Chronic Heart Failure, HTN: Hypertension Essential, CVA: Cerebral Vascular Event *P Value $<0.001$

\begin{tabular}{|c|c|c|c|c|}
\hline & $\begin{array}{c}\text { Group I } \\
\text { ARV }<=3.74\end{array}$ & $\begin{array}{c}\text { Group II } \\
3.75<A R V<4.6 \\
3\end{array}$ & $\begin{array}{c}\text { Group III } \\
4.64<\mathrm{ARV}<6.61\end{array}$ & $\begin{array}{l}\text { Group IV } \\
\text { ARV }>=5.62\end{array}$ \\
\hline Age * & $63.5 \pm 15.8$ & $66.3 \pm 13.1$ & $66.2 \pm 12.4$ & $68.3 \pm 11.7$ \\
\hline $\begin{array}{l}\text { Gender N(\%) } \\
\text { Male }\end{array}$ & $1199(62.5)$ & 1221(63.7) & $1224(63.8)$ & $1046(54.6)$ \\
\hline \multicolumn{5}{|l|}{ Surgery Type } \\
\hline Elective & $1564(83.4)$ & 1603(85.3) & 1632(86.7) & $1623(86.0)$ \\
\hline Emergency & $160(8.5)$ & $111(5.9)$ & $108(5.7)$ & $117(6.2)$ \\
\hline Urgent & 152(8.1) & $166(8.8)$ & $143(7.6)$ & $148(7.8)$ \\
\hline $\begin{array}{l}\text { Malignancy } \\
\mathrm{N}(\%)\end{array}$ & 718(37.5) & 654(34.1) & $518(27.0)$ & $476(24.8)$ \\
\hline $\begin{array}{l}\text { Renal Failure } \\
\mathbf{N}(\%)^{*}\end{array}$ & $427(22.3)$ & $347(18.1)$ & $305(15.9)$ & $284(14.8)$ \\
\hline Dialysis N(\%) & $80(4.2)$ & $46(2.4)$ & $44(2.3)$ & $34(1.8)$ \\
\hline IHD N(\%) & $275(14.3)$ & $380(19.8)$ & $477(24.9)$ & $570(29.7)$ \\
\hline CHF N(\%) * & $334(17.4)$ & $368(19.2)$ & $367(19.1)$ & $312(16.3)$ \\
\hline HTN N(\%) & $1273(66.4)$ & $1348(70.3)$ & $1387(72.4)$ & $1474(76.9)$ \\
\hline CVA N(\%) & $223(11.6)$ & $223(11.6)$ & 292(15.2) & $305(15.9)$ \\
\hline $\begin{array}{l}\text { Lung Disease } \\
\mathbf{N}(\%)\end{array}$ & $201(10.5)$ & $202(10.5)$ & $234(12.2)$ & $198(10.3)$ \\
\hline $\begin{array}{l}\text { Cirrhosis } \\
\mathrm{N}(\%)\end{array}$ & $23(1.2)$ & $12(0.6)$ & $13(0.7)$ & $13(0.7)$ \\
\hline
\end{tabular}

Table 2: Quartiles of mean blood pressure variability (ARV) in ascending order

\begin{tabular}{|l|c|c|c|c|}
\hline \multicolumn{5}{|c|}{ ARV-MAP Group } \\
\hline Group & I & II & III & IV \\
\hline Number & 1917 & 1917 & 1917 & 1917 \\
\hline ARV (mmHg/min) & $<=3.74$ & $\mathbf{3 . 7 5 - 4 . 6 3}$ & $\mathbf{4 . 6 4 - 5 . 6 1}$ & $>=5.62$ \\
\hline
\end{tabular}

Table 3: ARV and Postoperative Acute kidney injury (AKI) vs no Increase in postoperative creatinine value

*Gender, age, diabetes, CHF, cirrhosis, IHD, malignancy, smoking, CHF, HTN creatinine before operation

\begin{tabular}{|c|c|c|c|c|}
\hline & $\begin{array}{l}\text { ARV- } \\
\text { MIBP* }\end{array}$ & $\begin{array}{l}\text { Number } \\
\text { (Events) }\end{array}$ & Adjusted model*" & $\mathrm{p}$-value \\
\hline $\begin{array}{l}\text { Post operative AKI } \\
\text { according to KIDGO } \\
\text { staging((3) }\end{array}$ & $\begin{array}{l}\text { I } \\
\text { II } \\
\text { III } \\
\text { IV }\end{array}$ & $\begin{array}{l}1729(594) \\
1766(735) \\
1769(835) \\
1742(872)\end{array}$ & $\begin{array}{l}\text { Ref } \\
1.2(1.04-1.4) \\
1.42(1.23-1.64) \\
1.52(1.31-1.76)\end{array}$ & $\begin{array}{l}0.015 \\
<0.0001 \\
<0.0001\end{array}$ \\
\hline
\end{tabular}

Conclusions: There is an inverse relation between intraoperative $\mathrm{BP}$ variability and the risk for postoperative mortality (even when adjusted to various co morbidities and MBP). Variability appears to be an indicator of a balanced autonomic system and a preserved physiological reserve.

Some chronic disease states are associated with decreased BP variability (Malignancy, chronic renal failure and dialysis), While other are associated with increased BP variability (Ischemic heart disease, congestive heart failure and essential hypertension)

The risk for early post operative AKI, on the other hand, is directly related to intraoperative BP variability, irrespective of severity of AKI. This finding requires further validation and research. 